\title{
Inhaltsverzeichnis
}

\author{
Beiträge
}

Filipović, Emir O.: The Most Noble and Royal House of Kotromanić.

Constructing Dynastic Identity in Medieval Bosnia

Hristozov, Hristo: Overcrowding the Mountains in the Ottoman Balkans. Social and

Ecological Dimensions of the Demographic Crisis in the District ( $k a z a)$ of Nevrokop

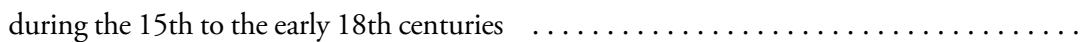

Dushku, Ledia: The Vilayet of Ioannina in Front of Hellenism $\ldots \ldots \ldots \ldots \ldots \ldots \ldots . \ldots 8$

Radovanović, Jelena: Can There Be a "Civilized Turk"? Encountering Ottoman Moder-

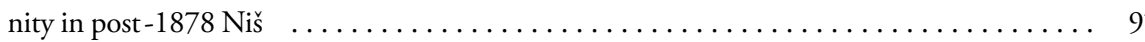

Papanikolaou, Konstantinos: Representations of Macedonia in German Public Discourse 1890-1912. From Colmar von der Goltz to Hans Friedrich Blunck

Ailianos, Costis J.: The Interaction between Greeks/Greece and Central/Southeastern

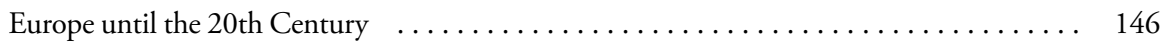

Mildenberger, Florian G. / Schwarz, Peter: Der Tanz in den Abgrund. Das Leben

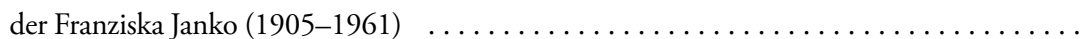

Bilogrivić, Goran: Medieval Reality or a Modern Construct? Old Croatian Culture in Medieval Studies Between the 19th and the 21st Century $\ldots \ldots \ldots \ldots \ldots \ldots \ldots$

\section{Aus der Südosteuropa-Forschung}

Ursinus, Michael: Cevdet Paşa und das Ahdname von Fojnica

Heppner, Harald / Kreuter, Peter Mario: Neue Studien zu Südosteuropa im 18. Jahrhundert

KIRCHNER, Hans-Martin (mit Ergänzungen von Konrad CLEWING): Warum Athen 1833 noch nicht Landeshauptstadt werden wollte. Ein Einblick in die vernachlässigten Protokolle des griechischen Regentschaftsrates

Istrate, Alexandru: Interroger l'histoire. Le questionnaire d'Alexandru Odobescu et son impact sur la mise en évidence des stratégies patrimoniales roumaines

KežIĆ,Danijel: Die Bedeutung einer Infrastrukturgeschichte Südosteuropas. Tendenzen und Desiderata in der aktuellen Ost- und Südosteuropaforschung

Heine, Martina: Der Brautzug der Erzherzogin Maria Christina von Graz nach Siebenbürgen im Jahre 1595. Ein Reisetagebuch $\ldots \ldots \ldots \ldots \ldots \ldots \ldots \ldots \ldots \ldots \ldots \ldots \ldots \ldots \ldots \ldots \ldots \ldots \ldots$

\section{Nachrufe}

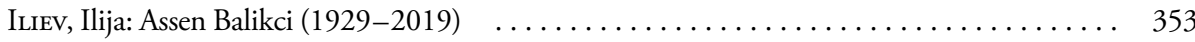

Matzinger, Joachim/Genesin, Monica: Wilfried Fiedler (1933-2019) … . . . . . 355 
Inhaltsverzeichnis

\section{Rezensionen}

Archive in Rumänien (I. und II.) (Andreas Becker)

BACHINGER, Bernhard: Die Mittelmächte an der Saloniki-Front 1915-1918. Zwischen

Zweck, Zwang und Zwist (Harald Potempa)

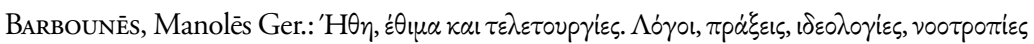

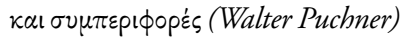

BARLETIUs, Marinus: De obsidione Scodrensi. Über die Belagerung von Skutari (Ina Arapi)

Bejan, Cristina A.: Intellectuals and Fascism in Interwar Romania. The Criterion Asso-

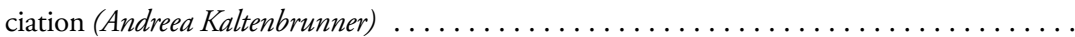

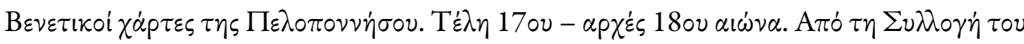

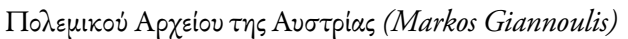

BITUNJAC, Martina: Verwicklung. Beteiligung. Unrecht. Frauen und die Ustaša-Bewegung (Sanela Schmid)

BRAUN, Bettina: Eine Kaiserin und zwei Kaiser. Maria Theresia und ihre Mitregenten Franz

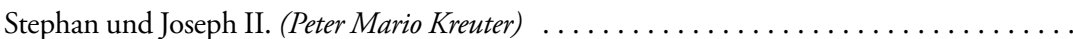

Center, Province and Periphery in the Age of Constantine VII Porphyrogennetos. From "De cerimoniis" to "De Administrando Imperio" (Predrag Komatina) . . . . . . . . . . . . . Clayer, Nathalie / Bougarel, Xavier: Europe’s Balkan Muslims. A New History (Ulf

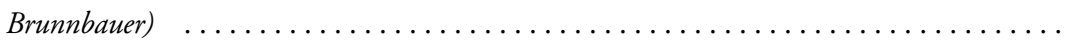

Corpus Draculianum. Dokumente und Chroniken zum walachischen Fürsten Vlad dem Pfähler 1448-1650. Band 1: Briefe und Urkunden aus der Walachei. Teil 1: Die Überlieferungen aus der Walachei (Bogdan Popa)

Diaconescu, Marius / Sora, Andrei Florin: Ziare românești despre Unirea Basarabiei, Bucovinei și Transilvaniei (Svetlana Suveica)

Die Deutschen in Rumänien 1944-1953. Eine Quellensammlung (Sven Schreiber).

Die getäuschte Wissenschaft. Ein Genie betrügt Europa - Konstantinos Simonides (Peter

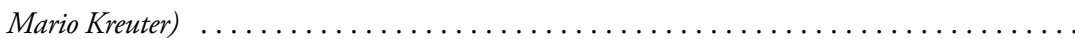

Die Kunst der Armenier im östlichen Europa (Alexander Zäh) $\ldots \ldots \ldots \ldots \ldots \ldots \ldots \ldots \ldots$

Die Verfolgung und Ermordung der europäischen Juden durch das nationalsozialistische

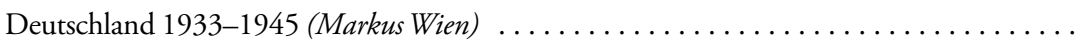

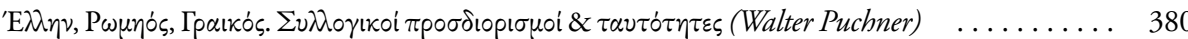

FiedLeR, Wilfried: Vergleichende Grammatik der Balkansprachen (Klaus Steinke) ．....... 568

Franz, Angelika/NösLer, Daniel: Geköpft und gepfählt. Archäologen auf der Jagd nach

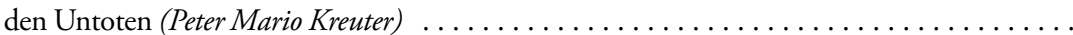

GeKas, Sakis: Xenocracy. State, Class and Colonialism in the Ionian Islands, 1815-1864

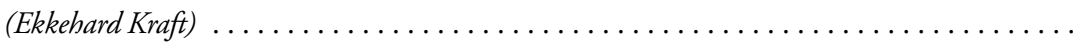

Himstedt-Vaid, Petra: Synkretistische Glaubensvorstellungen in den Volksliedern der

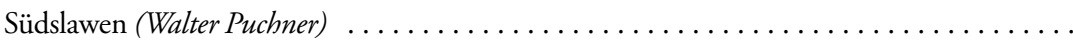


Inhaltsverzeichnis

Huber-Frischeis, Thomas/Knieling, Nina/Valenta, Rainer: Die Privatbibiothek Kaiser Franz' I. 1784-1835. Bibliotheks- und Kulturgeschichte einer fürstlichen Sammlung zwischen Aufklärung und Vormärz (Konrad Clewing) $\ldots \ldots \ldots \ldots \ldots \ldots \ldots$

Imagining Byzantium. Perceptions, Patterns, Problems (Alexander Zäh) $\ldots \ldots \ldots \ldots \ldots \ldots . \ldots \ldots 72$

Jareb, Mario: Mediji i promidžba u Nezavisnoj Državi Hrvatskoj (Marija Bitunjac) $\ldots \ldots \ldots 505$

KaHL, Thede: Natur und Mensch im Donaudelta (Walter Puchner) $\quad \ldots \ldots \ldots \ldots \ldots \ldots . \ldots . \ldots 39$

Keller, Markus: Experten und Beamte. Die Professionalisierung der Lehrer höherer Schulen in der zweiten Hälfte des 19. Jahrhunderts. Ungarn und Preußen im Ver-

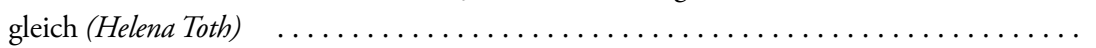

Kellmann, Klaus: Dimensionen der Mittäterschaft. Die europäische Kollaboration mit

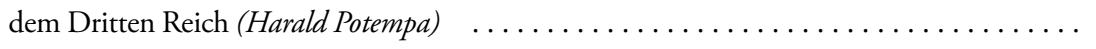

Kemalism. Transnational Politics in the Post-Ottoman World (Ellinor Morack) . . . . . . . 490

KurTović, Esad: Arhivska građa za istoriju srednjovjekovne Bosne. Ispisi iz knjiga kancelarije Državnog arhiva u Dubrovniku 1341-1526 (Oliver Jens Schmitt) ............

LaLIć, Daniel: Der Hochadel Kroatien-Slawoniens. Zwischen Verlust, Verteidigung und Neuerwerb gesellschaftlicher Elitenpositionen (1868-1918) (Wolfgang Kessler) .........

Lične zabilješke generala Oskara Potioreka o unutrašnjopolitičkoj situaciji u Bosni i

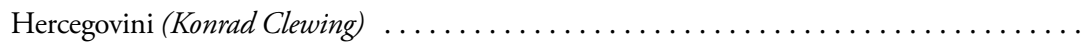

MARcıUc, Iuliana: Zei de mucava. Incursiuni imagologice în cinematografie şi media

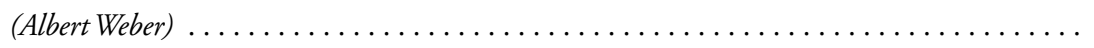

Marin, Irina: Peasant Violence and Antisemitism in Early Twentieth-Century Eastern

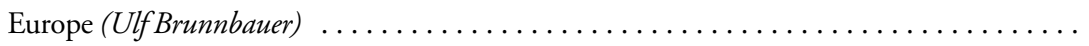

Mezarescu, Ion: Partidul Național Creștin. 1935-1938 (Andreea Kaltenbrunner). . . . . . . . . .

Mincić, Milan: Amerikanci. Srpski dobrovoljci iz SAD (1914-1918) (Ulf Brunn-

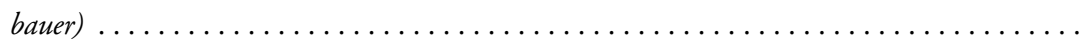

MolLA, Ylli: Guerilas Made in Albania. Historia e Arafatit, Kabilës, Lulës, Amazonas dhe luftëtarëve nga 11 shtete, që u përgatitën politikisht dhe ushtarakisht nga pedagogët

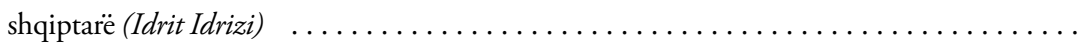

NeAgoe, Manole: Mistificări grosolane ale istoriei României. De la Mihai Roller la Neagu Djuvara și Lucian Boia (Peter Mario Kreuter) $\ldots \ldots \ldots \ldots \ldots \ldots \ldots \ldots \ldots \ldots \ldots \ldots \ldots \ldots \ldots \ldots \ldots \ldots$

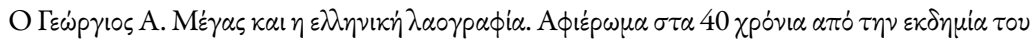

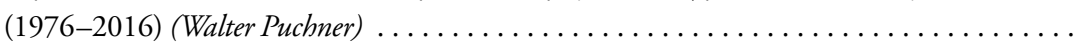

Pálosfalvi, Tamás: From Nicopolis to Mohács. A History of Ottoman-Hungarian War-

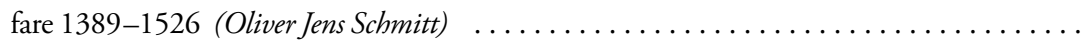

Pantelimon Halippa și problema Basarabiei în dosarele securității, 1965-1979 (Armin

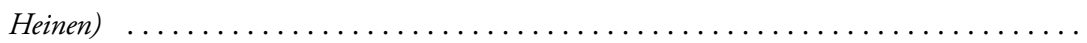

PÂrÂIANU, Răzvan: Octavian Goga. Sacerdote of the Nation Revisiting the Romanian

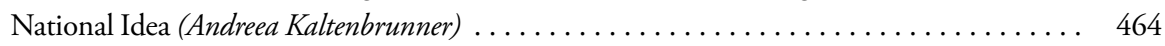

Peyrissac, Jean: En Macédoine sous la montagne bleue. Campagne d'Orient 1917-1918

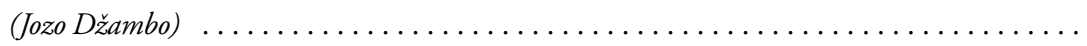


Pilat, Liviu/Cristea, Ovidiu: The Ottoman Threat and Crusading on the Eastern Border of Christendom during the $15^{\text {th }}$ Century (Oliver Jens Schmitt) $\ldots \ldots \ldots \ldots \ldots \ldots$

Prvovarenko, Aleksandr Aleksandrovič: Chorvatija. Istorija, politika, ideologija. Konec XX - načalo XXI veka (Ludwig Steindorff)

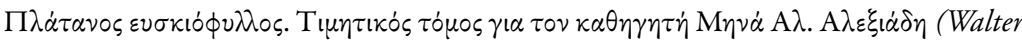
Puchner)

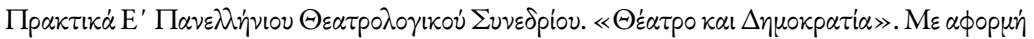

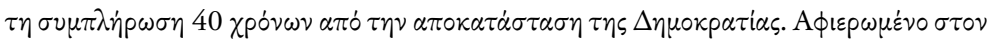

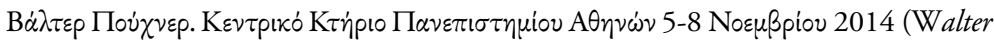
Puchner)

Rama 1968. Od iseljavanja do integracije. Međunarodni znanstveno-stručni skup (Jozo Džambo)

Richter, Heinz A.: Hellas und Zypern in meinem Leben. Erinnerungen eines Zeithisto-

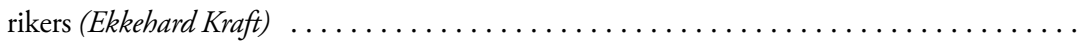

Richter, Heinz A.: Geschichte der griechischen Linken (Ekkehard Kraft) $\ldots \ldots \ldots \ldots \ldots$

Ristanović, Petar: Kosovsko pitanje 1974-1989 (Pieter Troch)

SABO, Klaudija: Ikonen der Nationen. Heldendarstellungen im post-sozialistischen Kroatien und Serbien (Frederik Lange)

Schumacher, Hubertus: Die k.u.k. Donauflottille im Ersten Weltkrieg. Karl Wettstein,

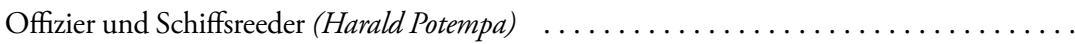

Seттsснек, Stefan: Die Tagebücher Kaiser Karls VI. Zwischen Arbeitseifer und Melancholie (Peter Mario Kreuter) . . . . . . . . . . . . . . . . . . . . . . . . . . . . .

SenjaKović, Reana: Svaki dan pobjeda. Kultura omladinskih radnih akcija (Danijel Kežić) . . . . . .

Skanderbeg - Rewizje, reprezentacje, reminiscencje (Ina Arapi) $\ldots \ldots \ldots \ldots \ldots \ldots \ldots \ldots 20$

TAKÁcs, Miklós: Byzantinische oder byzantinisierende Raumgestaltungen kirchlicher Architektur im frühárpádenzeitlichen Ungarn. Eine vergleichende Analyse auf der Grundlage von Parallelen aus dem Balkan (Alexander $Z \ddot{a h h) ~} \ldots \ldots \ldots \ldots \ldots \ldots$

TAMÁsKa, Maté: Armenian Townscapes in Transylvania (Alexander $Z \ddot{a h h) ~ \ldots . . . . . . . . . . . ~} 560$

TelbizovA-SACK, Jordanka: Islam in Europa. Transformation, religiöse Erneuerung und innere Diversifizierung am Beispiel Bulgariens (Xavier Bougarel) $\ldots \ldots \ldots \ldots \ldots \ldots$

Trauma und Erinnerung. Narrative Versionen zum Bürgerkrieg in Griechenland (Gustav Auernheimer)

Varvounis, Manolis G.: Traditional and Popular Religiosity in Greece. Customs and Rituals. Studies and Aspects on Social and Religious Folkloristics (Walter Puchner) . . . . . .

Verbündet. Bayern und Bulgarien im Ersten Weltkrieg. Eine Ausstellung des Bayerischen Hauptstaatsarchivs und der Staatsagentur „Archive“ Bulgariens (Markus Wien) . . . . . . . Verwaltetes Wasser im Österreich des Spätmittelalters und der frühen Neuzeit (Sebastian

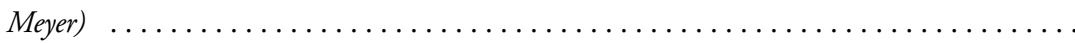

Vexierspiegel Securitate. Rumäniendeutsche Autoren im Visier des kommunistischen Geheimdienstes (Franziska Wagner) 
Inhaltsverzeichnis

Vlad der Pfähler - Dracula. Tyrann oder Volkstribun? (Bogdan Popa) $\ldots \ldots \ldots \ldots \ldots \ldots . \ldots 15$

VuČETIĆ, Radina: Monopol na istinu. Partija, kultura i cenzura u Srbiji šezdesetih i

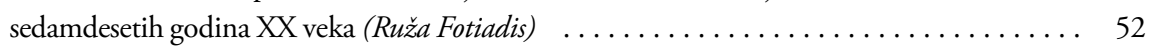

Zwischen Trauer und Triumph. Das Jahr 1918 in der mitteleuropäischen Literatur (Alice

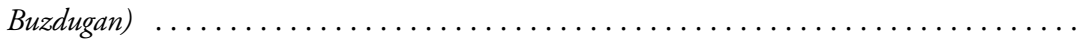

